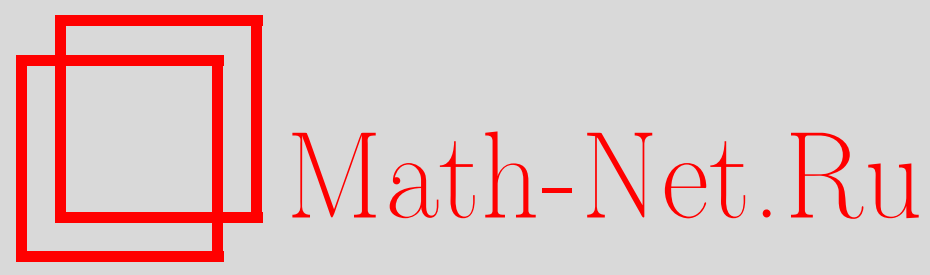

О. Н. Косухин, О скорости приближения замкнутых жордановых кривых, Матем. заметки, 2005, том 77, выпуск $6,861-876$

DOI: https://doi.org/10.4213/mzm2543

Использование Общероссийского математического портала Math-Net.Ru подразумевает, что вы прочитали и согласны с пользовательским соглашением http://www.mathnet.ru/rus/agreement

Параметры загрузки:

IP : 54.197 .217 .227

26 апреля 2023 г., 13:05:01

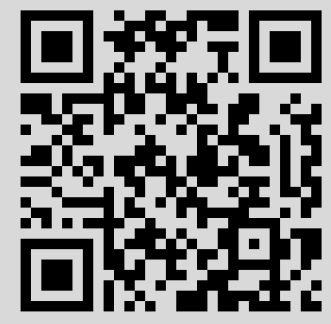




\section{О СКОРОСТИ ПРИБЛИЖЕНИЯ ЗАМКНУТЫХ ЖОРДАНОВЫХ КРИВЫХ ЛЕМНИСКАТАМИ}

\section{О.Н. Косухин}

Принципиальная возможность сколь угодно хорошего приближения произвольной замкнутой жордановой кривой $\Gamma$ на комплексной плоскости $\{z\}$ в метрике Хаусдорфа посредством лемнискат, порожденных многочленами $P(z)$, доказана Гильбертом. $\mathrm{B}$ настоящей работе получены количественные оценки сверху для наименьших уклонений $H_{n}(\Gamma)$ от кривой $Г$ (в этой метрике) лемнискат, порожденных многочленами заданной степени $n$, в терминах модулей непрерьвности конформного отображения внешности $Г$ на внешность единичной окружности, обратного к нему отображения и функции Грина с полюсом на бесконечности для внешности $\Gamma$. В случае аналитичности $\Gamma$ доказано, что $H_{n}(\Gamma)=O\left(q^{n}\right), 0 \leqslant q=q(\Gamma)<1, n \rightarrow \infty$.

Библиография: 6 названий.

Введение. В работе даются оценки сверху наименьших уклонений в метрике Хаусдорфа заданного замкнутого жорданова контура $\Gamma$ в комплексной плоскости $\mathbb{C}$ от лемнискат, порожденных многочленами $P(z)$ заданной степени $n$, в зависимости от метрических свойств этого контура. При этом предполагается, что приближающие лемнискаты являются замкнутьми жордановыми кривьми, содержащими Г внутри себя. Принципиальная возможность сколь угодно хорошего приближения жордановых контуров такими лемнискатами в этой метрике доказана Гильбертом в 1897 г. (см. [1]). Естественно возникает вопрос о количественной оценке такого приближения. Этот вопрос неоднократно ставил Е. П. Долженко.

В силу теорем Римана и Каратеодори существует единственное конформное и однолистное отображение $w=\psi(z)$ внешности $G_{\Gamma}$ кривой $\Gamma$ на внешность $G_{C}$ единичной окружности $C:=\{w:|w|=1\}$ со свойствами: $\psi(\infty)=\infty, \psi^{\prime}(\infty)>0$; отображение $w=\psi(z)$ продолжается до гомеоморфизма замыканий $\bar{G}_{\Gamma}$ и $\bar{G}_{C}$ областей $G_{\Gamma}$ и $G_{C}$. Положим

$$
\varphi(w):=\psi^{-1}(w), \quad w \in \bar{G}_{C}, \quad f(\theta):=\varphi\left(e^{i \theta}\right), \quad \theta \in \mathbb{R} .
$$

Пусть $A$ и $B$ - компакты на $\mathbb{C}, \rho_{H}(A, B)$ - хаусдорфово расстояние между ними: $\rho_{H}(A, B)$ $:=\inf \{\varepsilon\}$, где инфимум берется по всем таким $\varepsilon>0$, что множества $A$ и $B$ находятся в евклидовых $\varepsilon$-окрестностях друг друга. Через $\rho(A, B)$ обозначим обычное (евклидово) расстояние между $A$ и $B$ :

$$
\rho(A, B):=\inf \left\{\left|z_{1}-z_{2}\right|: z_{1} \in A, z_{2} \in B\right\} .
$$

Работа выполнена при поддержке Российского фонда фундаментальных исследований, грант № 02-01-00913, и программы “Ведущие научные школы” Президента РФ, грант № НШ-1892.2003.1. 
Пусть $P_{n}(z)$ - многочлен степени $n$ переменного $z, r=$ const $>0$,

$$
L\left(P_{n}, r\right):=\left\{z:\left|P_{n}(z)\right|=r\right\}
$$

- $r$-я лемниската, порожденная многочленом $P_{n}$, (т.е. его $r$-я линия уровня),

$$
H_{n}(\Gamma):=\inf \left\{\rho_{H}\left(\Gamma, L\left(P_{n}, r\right)\right): P_{n}, r\right\},
$$

где $P_{n}$ пробегает все многочлены степени $n$, а $r$ пробегает все положительные числа, причем учитьваются только те $P_{n}$ и $r$, для которых $L\left(P_{n}, r\right)$ является замкнутой жордановой кривой, содержащей Г внутри себя.

Пусть $g(z, \zeta)$ - функция Грина для области $G_{\Gamma}$ с полюсом $\zeta \in \bar{G}_{\Gamma}$. Как известно, $g(z, \infty)=\log |\psi(z)|=: g(z)$. Ниже

$$
\omega(F, E ; \delta):=\sup \left\{\left|F\left(z^{\prime}\right)-F\left(z^{\prime \prime}\right)\right|: z^{\prime}, z^{\prime \prime} \in E,\left|z^{\prime}-z^{\prime \prime}\right| \leqslant \delta\right\}
$$

- модуль непрерывности функции $F(z)$ на множестве $E \subset \mathbb{C}, \delta \geqslant 0$. Если множество $E$ есть вся область определения функции $F(z)$, положим $\omega(F ; \delta)=\omega(F, E ; \delta)$.

1. Для любого числа $\lambda \geqslant 0$ определим кривую

$$
\Gamma_{\lambda}:=\{z: g(z)=\lambda\} .
$$

Оценим величину

$$
M_{n}(\Gamma):=\inf \left\{\max \left\{g(z): z \in L\left(P_{n}, r\right)\right\}: P_{n}, r\right\},
$$

где $P_{n}$ и $r$ пробегают те же множества, что и выше при определении $H_{n}(\Gamma)$.

ЛЕмма. Для произвольной замкнутой жсордановой кривой $Г$ и любого натурального числа п выполнены неравенства

$$
H_{n}(\Gamma) \leqslant \omega\left(\varphi ; e^{M_{n}(\Gamma)}-1\right), \quad M_{n}(\Gamma) \leqslant \omega\left(g ; H_{n}(\Gamma)\right) .
$$

ДокАЗАТЕЛЬСТво. Пусть, как и ранее, многочлен $P_{n}$ и число $r>0$ таковы, что лемниската $L\left(P_{n}, r\right)$ является замкнутой жордановой аналитической кривой, содержашей Г внутри себя. Для произвольных таких $P_{n}$ и $r$ положим

$$
\lambda=\lambda\left(P_{n}, r\right):=\max \left\{g(z): z \in L\left(P_{n}, r\right)\right\} .
$$

Тогда

$$
\rho_{H}\left(\Gamma, L\left(P_{n}, r\right)\right) \leqslant \rho_{H}\left(\Gamma, \Gamma_{\lambda}\right) \leqslant \max \left\{\left|\varphi\left(e^{\lambda+i \theta}\right)-\varphi\left(e^{i \theta}\right)\right|: \theta \in \mathbb{R}\right\} \leqslant \omega\left(\varphi ; e^{\lambda}-1\right) .
$$

Переходя теперь в этом неравенстве к инфимуму (сначала слева, а затем справа) по всем допустимым $P_{n}$ и $r$, получаем первое из неравенств (1).

Очевидно, что $g(z) \leqslant \omega(g ; \rho(z, \Gamma))$. Поэтому имеем

$$
\max \left\{g(z): z \in L\left(P_{n}, r\right)\right\} \leqslant \omega\left(g ; \max \left\{\rho(z, \Gamma): z \in L\left(P_{n}, r\right)\right\}\right) \leqslant \omega\left(g ; \rho_{H}\left(\Gamma, L\left(P_{n}, r\right)\right)\right) .
$$

Переходя в этом неравенстве к инфимуму по всем допустимым $P_{n}$ и $r$, получаем второе из неравенств (1). Лемма доказана полностью.

Для кривой Г и любого натурального $n$ введем в рассмотрение многочлены $Q_{n}(z)$ и $\Phi_{n}(z)$. Положим

$$
Q_{n}(z):=c^{-n}\left(z-a_{1}\right)\left(z-a_{2}\right) \cdots\left(z-a_{n}\right),
$$

где $c=c(\Gamma)$ - гармоническая емкость кривой $\Gamma$ (см. [2, гл. $7, \S 3])$,

$$
a_{k}:=\varphi\left(\exp \left\{\frac{(2 k+1) \pi i}{n}\right\}\right) .
$$

Через $\Phi_{n}(z)$ обозначим многочлен $\Phi$ абера $n$-й степени, т.е. такой полином, что $\Phi_{n}(z)-$ $\psi^{n}(z)=O(1 / z)$ при $z \rightarrow \infty$ (см., например, [3, т. 2, гл. 5, п. 4.5]). 
ТЕОРема 1. Пусть Г - замкнутая жсорданова кривая, $g(z)=g(z, \infty)-$ функиия Грина с полюсом на бесконечности для внешности кривой Г. Тогда для любого натурального $n$ найдутся такие положительные числа $r_{1}=r_{1}(n)$ и $r_{2}=r_{2}(n)$, что лемнискаты $L\left(Q_{n}, r_{1}\right)$ и $L\left(\Phi_{n}, r_{2}\right)$ являются замкнутыми аналитическими жордановыми кривыми, содержащ,им Г внутри себя, и неравенство

$$
\max \left\{g(z): z \in L\left(Q_{n}, r_{1}\right)\right\} \leqslant \omega(g ; d)+2 \omega\left(\varphi, C ; \frac{\pi}{n}\right) \int_{d}^{\infty} \frac{\min \{\omega(\psi ; t), 2\}}{t^{2}} d t
$$

справедливо для любого $d \geqslant \omega(\varphi, C ; \pi / n)$, а неравенство

$$
\max \left\{g(z): z \in L\left(\Phi_{n}, r_{2}\right)\right\} \leqslant \omega(g ; d)+\omega\left(\varphi, C ; \frac{\pi}{n}\right) \int_{d}^{\infty} \frac{\min \{\omega(\psi ; t), 2\}}{t^{2}} d t
$$

справедливо для таких $d \geqslant \omega(\varphi, C ; \pi / n)$, что второе слагаемое справа в этом неравенстве не превосходит первого. Отсюда при таких же $d$ имеем

$$
M_{n}(\Gamma) \leqslant \omega(g ; d)+\omega\left(\varphi, C ; \frac{\pi}{n}\right) \int_{d}^{\infty} \frac{\min \{\omega(\psi ; t), 2\}}{t^{2}} d t .
$$

ЗАмЕчАниЕ. Указанные в теореме 1 условия справедливости неравенств (2)-(4) не существенны при их практическом применении, поскольку наилучшие по порядку величин оценки получаются при $d$, определяемых из условия равенства между собой двух слагаемых справа в этих неравенствах - так как при $d \searrow 0$ первое слагаемое справа убывает к нулю, второе возрастает к $+\infty$.

ДоКАЗАТЕЛЬСТво тЕОРЕМЫ 1 . Для функции $g(z)$ известно интегральное представление (см., например, [2, гл. $7, \S 3])$ :

$$
g(z)=\gamma+\frac{1}{2 \pi} \int_{\Gamma} \log |z-\zeta| d \operatorname{Arg} \psi(\zeta)
$$

где $\gamma=\gamma(\Gamma)=-\log c(\Gamma)-$ постоянная Робэна для кривой $\Gamma$, функция $\operatorname{Arg} \psi(z)=$ $\operatorname{Im} \log \psi(z)$ является гармонически сопряженной к $g(z)$ (каждая ее локально непрерывная ветвь на $\Gamma$ строго возрастает и получает приращение $2 \pi$ при однократном обходе $\Gamma$ в положительном направлении). Отсюда при $f(\theta)=\varphi\left(e^{i \theta}\right)$ получаем представление

$$
g(z)=\gamma+\frac{1}{2 \pi} \int_{0}^{2 \pi} \log |z-f(\theta)| d \theta .
$$

Для каждого $k=0,1, \ldots, n-1$ положим

$$
\theta_{k}:=\frac{(2 k+1) \pi}{n}=\frac{1}{2}\left(\frac{2 \pi k}{n}+\frac{2 \pi(k+1)}{n}\right) .
$$

Тогда $e^{i \theta_{k}} \in C, f\left(\theta_{k}\right) \in \Gamma$. При любом $z \in G_{\Gamma}$ имеем для

$$
S:=\left|g(z)-\left(\gamma+\frac{1}{n} \sum_{k=1}^{n} \log \left|z-f\left(\theta_{k}\right)\right|\right)\right|
$$


следующее выражение:

$$
\begin{aligned}
S & =\left|\frac{1}{2 \pi} \int_{0}^{2 \pi} \log \right| z-f(\theta)\left|d \theta-\frac{1}{2 \pi} \sum_{k=1}^{n} \frac{2 \pi}{n} \log \right| z-f\left(\theta_{k}\right)|| \\
& =\left|\frac{1}{2 \pi} \sum_{k=0}^{n-1} \int_{2 \pi k / n}^{2 \pi(k+1) / n}\left(\log |z-f(\theta)|-\log \left|z-f\left(\theta_{k}\right)\right|\right) d \theta\right|
\end{aligned}
$$

Для любых наперед заданных положительных чисел $r_{0}$ и $r$ по формуле Тейлора для функции $\log r$ с остаточным членом в форме Лагранжа получаем равенство

$$
\log r=\log r_{0}+\frac{\left(r-r_{0}\right)}{r^{*}}
$$

где $r^{*}>0$ заключено между числами $r_{0}$ и $r$. Полагая $r=|z-f(\theta)|, r_{0}=\left|z-f\left(\theta_{k}\right)\right|$, $k=0,1, \ldots, n-1$, отсюда получаем

$$
\begin{aligned}
S & \leqslant \frac{1}{2 \pi} \sum_{k=0}^{n-1} \int_{2 \pi k / n}^{2 \pi(k+1) / n} \frac{|| z-f(\theta)|-| z-f\left(\theta_{k}\right)|| d \theta}{\left|z-f\left(\theta_{k}^{*}\right)\right|} \\
& \leqslant \frac{1}{2 \pi} \sum_{k=0}^{n-1} \int_{2 \pi k / n}^{2 \pi(k+1) / n} \frac{\left|f(\theta)-f\left(\theta_{k}\right)\right| d \theta}{\left|z-f\left(\theta_{k}^{*}\right)\right|} .
\end{aligned}
$$

Здесь $\theta_{k}^{*}=\theta_{k}^{*}(\theta)$ - такое число, лежащее между $\theta$ и $\theta_{k}$, что $r^{*}=r^{*}(k, \theta)=\left|z-f\left(\theta_{k}^{*}\right)\right|$.

Пусть далее при рассматриваемом $n$ точка $z \in \bar{G}_{\Gamma} \backslash\{\infty\}$ удовлетворяет условию $d^{*}:=\rho(z, \Gamma) \geqslant \omega(\varphi, C ; \pi / n)$. Тогда

$$
|z-f(\theta)| \leqslant\left|z-f\left(\theta_{k}^{*}\right)\right|+\left|f\left(\theta_{k}^{*}\right)-f(\theta)\right| \leqslant\left|z-f\left(\theta_{k}^{*}\right)\right|+\omega\left(\varphi, C ; \frac{\pi}{n}\right) \leqslant 2\left|z-f\left(\theta_{k}^{*}\right)\right|
$$

и, следовательно, $1 /\left|z-f\left(\theta^{*}\right)\right| \leqslant 2 /|z-f(\theta)|$. Поэтому из (7) получаем

$$
S \leqslant \frac{1}{\pi} \sum_{k=0}^{n-1} \int_{2 \pi k / n}^{2 \pi(k+1) / n} \frac{\left|f(\theta)-f\left(\theta_{k}\right)\right|}{|z-f(\theta)|} d \theta \leqslant \frac{\omega(\varphi, C ; \pi / n)}{\pi} \int_{0}^{2 \pi} \frac{d \theta}{|z-f(\theta)|}
$$

Пусть $m(t):=\sup \left\{\operatorname{mes}\left[\psi\left(B_{t} \cap \Gamma\right)\right]\right\}$, где супремум берется по всем замкнутьм кругам $B_{t}$ радиуса $t$, центры которых лежат в области $G_{\Gamma}$, a mes - линейная мера на окружности $C$. Оценим интеграл в правой части неравенства $(8)$ :

$$
\begin{aligned}
\int_{0}^{2 \pi} \frac{d \theta}{|z-f(\theta)|} & \left.\left.=\int_{0}^{\infty} \frac{1}{t} d \operatorname{mes}\{\theta:|z-f(\theta)| \leqslant t)\right\}=\int_{d^{*}}^{\infty} \frac{1}{t} d \operatorname{mes}\{\theta:|z-f(\theta)| \leqslant t)\right\} \\
& =-\frac{\operatorname{mes}\left\{\theta:|z-f(\theta)| \leqslant d^{*}\right\}}{d^{*}}+\int_{d^{*}}^{\infty} \frac{\operatorname{mes}\{\theta:|z-f(\theta)| \leqslant t\}}{t^{2}} d t \\
& \leqslant \int_{d^{*}}^{\infty} \frac{\operatorname{mes}\{\theta:|z-f(\theta)| \leqslant t)\}}{t^{2}} d t \leqslant \int_{d^{*}}^{\infty} \frac{m(t)}{t^{2}} d t
\end{aligned}
$$


Отсюда и из неравенства (8) получаем

$$
S=\left|g(z)-\left(\gamma+\left(\frac{1}{n}\right) \sum_{k=1}^{n} \log \left|z-f\left(\theta_{k}\right)\right|\right)\right| \leqslant \frac{\omega(\varphi, C ; \pi / n)}{\pi} \int_{d^{*}}^{\infty} \frac{m(t)}{t^{2}} d t
$$

для любых таких $z \in \bar{G}_{\Gamma} \backslash\{\infty\}$, что $d^{*}=\rho(z, \Gamma) \geqslant \omega(\varphi, C ; \pi / n)$. Напомним, что $Q_{n}(z):=c^{-n}\left(z-a_{1}\right)\left(z-a_{2}\right) \cdots\left(z-a_{n}\right)$, где $c=c(\Gamma)=e^{-\gamma}-$ гармоническая емкость кривой $\Gamma, a_{k}:=f\left(\theta_{k-1}\right)=\varphi\left(e^{i \theta_{k-1}}\right), k=1,2, \ldots, n$. Домножая обе части неравенства (9) на $n$ и потенцируя, получаем

$$
\begin{aligned}
\exp \left(-\frac{n}{\pi} \omega\left(\varphi, C ; \frac{\pi}{n}\right) \int_{d^{*}}^{\infty} \frac{m(t)}{t^{2}} d t\right) & \leqslant \frac{\exp \{n g(z)\}}{\left|Q_{n}(z)\right|}=\frac{\left|\psi^{n}(z)\right|}{\left|Q_{n}(z)\right|} \\
& \leqslant \exp \left(\frac{n}{\pi} \omega\left(\varphi, C ; \frac{\pi}{n}\right) \int_{d^{*}}^{\infty} \frac{m(t)}{t^{2}} d t\right)
\end{aligned}
$$

Возьмем $\lambda>0$ таким, чтобы было

$$
d^{* *}=d^{* *}(\lambda):=\rho\left(\Gamma, \Gamma_{\lambda}\right)=\min \left\{d^{*}=\rho(z, \Gamma): z \in \Gamma_{\lambda}\right\} \geqslant \omega\left(\varphi, C ; \frac{\pi}{n}\right)
$$

(так что при $z \in \bar{G}_{\Gamma_{\lambda}}$ выполняется принятое вьше условие $\rho(z, \Gamma) \geqslant \omega(\varphi, C ; \pi / n)$ ), и положим $r_{1}=\max \left\{\left|Q_{n}(z)\right|: z \in \Gamma_{\lambda}\right\}$. Напомним, что $\Gamma_{\lambda}:=\{z: g(z)=\lambda\}, \rho\left(\Gamma, \Gamma_{\lambda}\right)-$ обычное (евклидово) расстояние между $Г$ и $\Gamma_{\lambda}$. Тогда по левому из неравенств (10) имеем при $z \in \Gamma_{\lambda}$

$$
\begin{gathered}
\exp \left(-\frac{n}{\pi} \omega\left(\varphi, C ; \frac{\pi}{n}\right) \int_{d^{* *}}^{\infty} \frac{m(t)}{t^{2}} d t\right) \leqslant \frac{\exp \{n \lambda)\}}{\left|Q_{n}(z)\right|} \\
r_{1}=\max \left\{\left|Q_{n}(z)\right|: z \in \Gamma_{\lambda}\right\} \leqslant \exp \left(n\left(\lambda+\frac{\omega(\varphi, C ; \pi / n)}{\pi} \int_{d^{* *}}^{\infty} \frac{m(t)}{t^{2}} d t\right)\right) .
\end{gathered}
$$

Используя это неравенство, из правого неравенства в (10) аналогично получаем при $z \in L\left(Q_{n}, r_{1}\right)$ :

$$
\begin{aligned}
\exp \{n g(z)\} & \leqslant\left|Q_{n}(z)\right| \exp \left(\frac{n}{\pi} \omega\left(\varphi, C ; \frac{\pi}{n}\right) \int_{d^{*}}^{\infty} \frac{m(t)}{t^{2}} d t\right) \\
& =r_{1} \exp \left(\frac{n}{\pi} \omega\left(\varphi, C ; \frac{\pi}{n}\right) \int_{d^{*}}^{\infty} \frac{m(t)}{t^{2}} d t\right) \\
& \leqslant \exp \left(n\left(\lambda+\frac{2 \omega(\varphi, C ; \pi / n)}{\pi} \int_{d^{* *}}^{\infty} \frac{m(t)}{t^{2}} d t\right)\right), \\
\max \{g(z): z & \left.\in L\left(Q_{n}, r\right)\right\} \leqslant \lambda+\frac{2 \omega(\varphi, C ; \pi / n)}{\pi} \int_{d^{* *}}^{\infty} \frac{m(t)}{t^{2}} d t .
\end{aligned}
$$

Иными словами, лемниската $L=L\left(Q_{n}, r_{1}\right)$ заключена между аналитическими контурами $\Gamma_{\lambda}$ и $\Gamma_{\lambda+\varepsilon}$, где

$$
\varepsilon=\frac{2}{\pi} \omega\left(\varphi, C ; \frac{\pi}{n}\right) \int_{d^{* *}}^{2 \pi} \frac{m(t)}{t^{2}} d t
$$


Так как все нули $\left\{a_{k}\right\}$ многочлена $Q_{n}(z)$ лежат на кривой $\Gamma$, то они лежат в одной и той же компоненте связности дополнения к лемнискате $L$. Поэтому $L$ является замкнутой жордановой аналитической кривой. Возьмем какое-нибудь число $d \geqslant \omega(\varphi, C ; \pi / n)$ и положим $\lambda=\omega(g ; d)$. Тогда $\omega(g ; d)=\lambda \leqslant \omega\left(g ; \rho\left(\Gamma, \Gamma_{\lambda}\right)\right)=\omega\left(g ; d^{* *}(\lambda)\right)$ и, следовательно, $d \leqslant d^{* *}$ (таким образом, при $z \in \bar{G}_{\Gamma_{\lambda}}$ вьполнены условия, наложенные вьше на $z$ ). Для завершения доказательства неравенства (2) заметим, что для любого $t>0$ имеем $m(t) \leqslant \min \{\pi \omega(\psi ; t), 2 \pi\}$.

Перейдем теперь к доказательству второго неравенства. Легко показать (см., например, [3, т. 2 , гл. 5 , п. 4.5]), что для любого $\varepsilon>0$ и всех $z \in G_{\Gamma_{\varepsilon}}$ имеет место равенство

$$
\Phi_{n}(z)-\psi^{n}(z)=\frac{1}{2 \pi i} \int_{\Gamma_{\varepsilon}} \frac{\psi^{n}(\zeta)}{\zeta-z} d \zeta
$$

Пусть $\log (\zeta-z)$ - некоторая аналитическая на кривой $\Gamma_{\varepsilon}$ ветвь функции $\log (\zeta-z)$. Пусть $\theta \in[0,2 \pi]$, тогда равенство $\theta=\varphi\left(e^{\varepsilon+i \theta}\right)$ определяет параметризацию кривой $\Gamma_{\varepsilon}$. Имеем $\psi^{n}(\zeta)=e^{n(\varepsilon+i \theta)}$. Преобразуя интеграл в правой части равенства $(11)$, получаем

$$
\begin{aligned}
\Phi_{n}(z)-\psi^{n}(z) & =\frac{1}{2 \pi i} \int_{\Gamma_{\varepsilon}} \frac{\psi^{n}(\zeta)}{\zeta-z} d \zeta=\frac{1}{2 \pi i} \int_{\Gamma_{\varepsilon}} \psi^{n}(\zeta) d \log (\zeta-z) \\
& =-\frac{1}{2 \pi i} \int_{\Gamma_{\varepsilon}} \log (\zeta-z) d \psi^{n}(\zeta)=-\frac{e^{n \varepsilon}}{2 \pi i} \int_{0}^{2 \pi} \log \left(\varphi\left(e^{\varepsilon+i \theta}\right)-z\right) d e^{i n \theta} \\
& =-\frac{n e^{n \varepsilon}}{2 \pi} \int_{0}^{2 \pi} \log \left(\varphi\left(e^{\varepsilon+i \theta}\right)-z\right) e^{i n \theta} d \theta \\
& =\frac{n e^{n \varepsilon}}{4 \pi} \int_{0}^{2 \pi}\left(\log \left(\varphi\left(e^{\varepsilon+i(\theta+\pi / n)}\right)-z\right)-\log \left(\varphi\left(e^{\varepsilon+i \theta}\right)-z\right)\right) e^{i n \theta} d \theta
\end{aligned}
$$

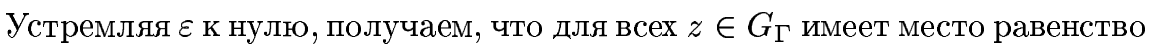

$$
\Phi_{n}(z)-\psi^{n}(z)=\frac{n}{4 \pi} \int_{0}^{2 \pi}(\log (f(\theta+\pi / n)-z)-\log (f(\theta)-z)) e^{i n \theta} d \theta
$$

При $d^{*}=\rho(z, \Gamma) \geqslant \omega(\varphi, C ; \pi / n)$ аналогично рассуждениям, предшествуюшим неравенствам (8), получаем оценку сверху

$$
\left|\log \left(f\left(\theta+\frac{\pi}{n}\right)-z\right)-\log (f(\theta)-z)\right| \leqslant 2 \frac{\omega(\varphi, C ; \pi / n)}{|z-f(\theta)|} .
$$

Отсюда следует

$$
\left|\Phi_{n}(z)-\psi^{n}(z)\right| \leqslant \frac{n \omega(\varphi, C ; \pi / n)}{2 \pi} \int_{0}^{2 \pi} \frac{1}{|z-f(\theta)|} d \theta \leqslant \frac{n \omega(\varphi, C ; \pi / n)}{2 \pi} \int_{d^{*}}^{\infty} \frac{m(t)}{t^{2}} d t .
$$

Имеем

$$
|\log | \Phi_{n}(z)|-\log | \psi^{n}(z)|| \leqslant \frac{\left|\Phi_{n}(z)-\psi^{n}(z)\right|}{\min \left\{\left|\Phi_{n}(z)\right|,\left|\psi^{n}(z)\right|\right\}} \leqslant \frac{\left|\Phi_{n}(z)-\psi^{n}(z)\right|}{\left(\left|\psi^{n}(z)\right|-\left|\Phi_{n}(z)-\psi^{n}(z)\right|\right)} .
$$


Пусть $|\psi(z)|=e^{\lambda}$. Тогда при

$$
\lambda \geqslant \frac{\omega(\varphi, C ; \pi / n)}{2 \pi} \cdot \int_{d^{*}}^{\infty} \frac{m(t)}{t^{2}} d t
$$

справедливы неравенства

$$
\left|\psi^{n}(z)\right|-\left|\Phi_{n}(z)-\psi^{n}(z)\right| \geqslant 1+n \lambda-\frac{n \omega(\varphi, C ; \pi / n)}{2 \pi} \int_{d^{*}}^{\infty} \frac{m(t)}{t^{2}} d t \geqslant 1 .
$$

Поэтому при указанньх условиях на $\lambda$ имеем $|\log | \psi^{n}(z)|-\log | \Phi_{n}(z)|| \leqslant\left|\psi^{n}(z)-\Phi_{n}(z)\right|$. То есть

$$
\exp \left(-n \frac{\omega(\varphi, C ; \pi / n)}{2 \pi} \int_{d^{*}}^{\infty} \frac{m(t)}{t^{2}} d t\right) \leqslant \frac{\left|\psi^{n}(z)\right|}{\left|\Phi_{n}(z)\right|} \leqslant \exp \left(n \frac{\omega(\varphi, C ; \pi / n)}{2 \pi} \int_{d^{*}}^{\infty} \frac{m(t)}{t^{2}} d t\right) .
$$

Повторяя рассуждения из первой части доказательства теоремы, следующие за неравенствами $(10)$, уже для многочлена $\Phi_{n}(z)$ получаем, что

$$
\max \left\{g(z): z \in L\left(\Phi_{n}, r_{2}\right)\right\} \leqslant \omega(g ; d)+\omega\left(\varphi, C ; \frac{\pi}{n}\right) \int_{d}^{\infty} \frac{\min \{\omega(\psi ; t), 2\}}{t^{2}} d t
$$

для некоторого положительного числа $r_{2}=r_{2}(n)$ и для любого $d \geqslant \omega(\varphi, C ; \pi / n)$, при котором второе слагаемое справа в этом неравенстве не превосходит первого (тогда для $\lambda=\omega(g ; d)$ вьполнены налагаемые на него вьше условия), причем лемниската $L\left(\Phi_{n}, r_{2}\right)$ является замкнутой аналитической жордановой кривой, содержащей Г внутри себя. Теорема доказана полностью.

СлЕДСТВИЕ 1. Для любой замкнутой жмордановой кривой Г имеет место равенство

$$
M_{n}(\Gamma)=O\left(\sqrt[3]{\omega\left(\varphi, C ; \frac{\pi}{n}\right)}\right), \quad n \rightarrow \infty
$$

ДокАЗАТЕЛЬСтво. Заметим, что $\omega(g ; d)=O(\sqrt{d})$ при $d \rightarrow 0, d>0$. Это следует, например, из теоремы 3 работы Тамразова [4]. Далее, $\omega(\psi ; t)=o(1)$ при $t \rightarrow 0, t>0$, и, следовательно,

$$
\int_{d}^{\infty} \frac{\min \{\omega(\psi ; t), 2\}}{t^{2}} d t=o\left(\frac{1}{d}\right) \quad \text { при } d \rightarrow 0, d>0 .
$$

Положим $d=\sqrt[3]{\omega^{2}(\varphi, C ; \pi / n)}$. Тогда

$$
\begin{gathered}
\omega(g ; d)=O\left(\sqrt[3]{\omega\left(\varphi, C ; \frac{\pi}{n}\right)}\right) \\
\omega\left(\varphi, C ; \frac{\pi}{n}\right) \cdot \int_{d}^{\infty} \frac{\min \{\omega(\psi ; t), 2\}}{t^{2}} d t=O\left(\sqrt[3]{\omega\left(\varphi, C ; \frac{\pi}{n}\right)}\right)
\end{gathered}
$$

По теореме 1 получаем требуемое равенство. 
ЗАмЕЧАнИЕ. Из следствия 1 вытекает, что для любой замкнутой жордановой кривой $\Gamma$ имеем $M_{n}(\Gamma) \rightarrow 0$ при $n \rightarrow \infty$. По лемме получаем, что $H_{n}(\Gamma) \rightarrow 0$ при $n \rightarrow \infty$. В этом и состоит упомянутый вьше результат Гильберта.

СледСтвиЕ 2. Пусть Г-замкнутая жсорданова кривая, функиия $\psi$ удовлетворяет в $\bar{G}_{\Gamma}$ условию $\operatorname{Lip} \alpha, 0<\alpha \leqslant 1$.

$$
\left|\psi\left(z^{\prime}\right)-\psi\left(z^{\prime \prime}\right)\right| \leqslant M\left|z^{\prime}-z^{\prime \prime}\right|^{\alpha}
$$

для любых $z^{\prime}, z^{\prime \prime}$ из $\bar{G}_{\Gamma}, M=\mathrm{const}>0$. Тогда при $0<\alpha<1$ выполнено соотношение

$$
M_{n}(\Gamma)=O\left(\omega^{\beta}\left(\varphi, C ; \frac{\pi}{n}\right)\right), \quad n \rightarrow \infty,
$$

əде $\beta=\max \{\alpha, 1 /(3-2 \alpha)\}$, a при $\alpha=1$

$$
M_{n}(\Gamma)=O\left(\omega\left(\varphi, C ; \frac{\pi}{n}\right) \log \left(\frac{1}{\omega(\varphi, C ; \pi / n)}\right)\right), \quad n \rightarrow \infty .
$$

ДокаЗАТЕЛЬСтво. Пусть сначала $0<\alpha<1$. Заметим, что если функция $\psi(z)$ удовлетворяет в $\bar{G}_{\Gamma}$ условию $\operatorname{Lip} \alpha$, то и функция $g(z)$ удовлетворяет в $\bar{G}_{\Gamma}$ этому условию:

$$
\begin{aligned}
\left|g\left(z^{\prime}\right)-g\left(z^{\prime \prime}\right)\right| & =|\log | \psi\left(z^{\prime}\right)|-\log | \psi\left(z^{\prime \prime}\right)|| \leqslant|| \psi\left(z^{\prime}\right)|-| \psi\left(z^{\prime \prime}\right)|| \\
& \leqslant\left|\psi\left(z^{\prime}\right)-\psi\left(z^{\prime \prime}\right)\right| \leqslant M\left|z^{\prime}-z^{\prime \prime}\right|^{\alpha}
\end{aligned}
$$

для любых $z^{\prime}$ и $z^{\prime \prime}$ из $\bar{G}_{\Gamma}$. Следовательно, для любого числа $d>0$ получаем неравенство $\omega(g ; d) \leqslant M d^{\alpha}$. Имеем также $\omega(\psi ; t) / t^{2} \leqslant M t^{\alpha-2}$ для любого $t>0$. Поэтому

$$
\int_{d}^{\infty} \frac{\min \{\omega(\psi ; t), 2\}}{t^{2}} d t=O\left(d^{\alpha-1}\right) .
$$

Положим $d=\omega(\varphi, C ; \pi / n)$. Тогда по теореме 1 получаем, что

$$
\begin{aligned}
M_{n}(\Gamma) & \leqslant M \omega\left(\varphi, C ; \frac{\pi}{n}\right)^{\alpha}+\omega\left(\varphi, C ; \frac{\pi}{n}\right) O\left(\omega^{\alpha-1}\left(\varphi, C ; \frac{\pi}{n}\right)\right) \\
& =O\left(\omega^{\alpha}\left(\varphi, C ; \frac{\pi}{n}\right)\right) \quad \text { при } n \rightarrow \infty .
\end{aligned}
$$

Заметим, что $\beta=\alpha$ при $1 / 2<\alpha<1$ и $\beta=1 /(3-2 \alpha)$ при $0<\alpha \leqslant 1 / 2$. В первом случае требуемое соотношение доказано. Рассмотрим случай $0<\alpha \leqslant 1 / 2$. Тогда $2 /(3-2 \alpha) \leqslant 1$. Как уже было отмечено вьше, для любой кривой $\Gamma$ имеем $\omega(g ; d)=O(\sqrt{d})$ при $d \rightarrow 0, d>0$. То есть сушествует такая постоянная $k=k(\Gamma)$, что при достаточно малых $d>0$ справедливо неравенство $\omega(g ; d) \leqslant k \sqrt{d}$. Положим $d=\omega^{2 /(3-2 \alpha)}(\varphi, C ; \pi / n)$. Тогда

$$
\int_{d}^{\infty} \frac{\min \{\omega(\psi ; t), 2\}}{t^{2}} d t=O\left(d^{\alpha-1}\right)=O\left(\omega^{2(\alpha-1) /(3-2 \alpha)}\left(\varphi, C ; \frac{\pi}{n}\right)\right) .
$$


Легко видеть, что $1+2(\alpha-1) /(3-2 \alpha)=1 /(3-2 \alpha)$. По теореме 1 получаем $M_{n}(\Gamma) \leqslant$ $O\left(\omega^{1 /(3-2 \alpha)}(\varphi, C ; \pi / n)\right)$.

Пусть теперь $\alpha=1$. Тогда $\omega(g ; d) \leqslant M d, \omega(\psi ; d) \leqslant M d$ для любого $d>0$. Поэтому

$$
\int_{d}^{\infty} \frac{\min \{\omega(\psi ; t), 2\}}{t^{2}} d t=O(-\log d) \quad \text { при } \quad d \rightarrow 0
$$

Полагая $d=\omega(\varphi, C ; \pi / n)$, по теореме 1 получаем требуемое соотношение.

Для того чтобы получить оценку величин $H_{n}(\Gamma)$ и $M_{n}(\Gamma)$ непосредственно в терминах метрических свойств кривой $\Gamma$, можно воспользоваться результатами работы Долженко [5], в которой дается оценка сверху на модули непрерьвности функций $\hat{\psi}, \hat{\varphi}-$ соответственно однолистного конформного отображения внутренности замкнутой жордановой кривой $\widehat{\Gamma}$ на внутренность единичного круга и обратного к нему отображения в метрических терминах кривой $\widehat{\Gamma}$, если определить $\widehat{\Gamma}$ как образ кривой Г при отображении $\hat{z}=1 /(z-a)$, где $a-$ некоторая точка, лежащая внутри кривой $\Gamma$.

2. ТЕОрема 2. Пусть Г - гладкая замкнутая жсорданова кривая, причем функиии $\psi(z)$ и $\varphi(w)$ непрерывно дифференцируемы в $\bar{G}_{\Gamma} u \bar{G}_{C}$ соответственно; кроме mozo,

$\omega\left(\arg f^{\prime}, \mathbb{R} ; \delta\right)=O\left(\frac{1}{\log (1 / \delta)}\right), \quad \omega\left(f^{\prime}, \mathbb{R} ; \delta\right)=O\left(\frac{1}{\log (1 / \delta)}\right) \quad n p u \quad \delta \rightarrow 0, \quad \delta>0$.

Тогда существуют такие числа $r_{1}=r_{1}(n)>0$ u $r_{2}=r_{2}(n)>0$, что при $n \rightarrow \infty$ имеют место равенства

$$
\max \left\{g(z): z \in L\left(Q_{n}, r_{1}\right)\right\}=O\left(\frac{1}{n}\right), \quad \max \left\{g(z): z \in L\left(\Phi_{n}, r_{2}\right)\right\}=O\left(\frac{1}{n}\right),
$$

так что (см. лемму

$$
H_{n}(\Gamma)=O\left(\frac{1}{n}\right), \quad M_{n}(\Gamma)=O\left(\frac{1}{n}\right), \quad n \rightarrow \infty .
$$

ДокАЗАТЕЛЬСтво. Пусть, как и ранее, $\theta_{k}:=(2 k+1) \pi / n$ для любого $k=0,1, \ldots$, $n-1$. Пусть также $z \in G_{\Gamma}$ и $\rho(z, \Gamma) \geqslant \omega(\varphi, C ; \pi / n)$. Для любых наперед заданных положительных чисел $r_{0}$ и $r$ имеет место формула Тейлора для функции $\log r$ с остаточным членом в форме Лагранжа:

$$
\log r=\log r_{0}+\frac{\left(r-r_{0}\right)}{r_{0}}-\frac{\left(r-r_{0}\right)^{2}}{2 r^{* 2}}
$$

где $r^{*}>0$ заключено между числами $r_{0}$ и $r$. Применим эту формулу к подьнтегральным функциям в сумме

$$
\sum_{k=1}^{n}\left|\int_{2 \pi k / n}^{2 \pi(k+1) / n}\left(\log |z-f(\theta)|-\log \left|z-f\left(\theta_{k}\right)\right|\right) d \theta\right|
$$


полагая в $k$-м ее члене $r_{0}=\left|z-f\left(\theta_{k}\right)\right|, r=|z-f(\theta)|$ при всех $k=0,1, \ldots, n-1$. Имеем

$$
\begin{aligned}
& \sum_{k=1}^{n}\left|\int_{2 \pi k / n}^{2 \pi(k+1) / n}\left(\log |z-f(\theta)|-\log \left|z-f\left(\theta_{k}\right)\right|\right) d \theta\right| \\
& \quad=\sum_{k=1}^{n}\left|\int_{2 \pi k / n}^{2 \pi(k+1) / n}\left(\frac{\left(|z-f(\theta)|-\left|z-f\left(\theta_{k}\right)\right|\right)}{\left|z-f\left(\theta_{k}\right)\right|}-\frac{\left(|z-f(\theta)|-\left|z-f\left(\theta_{k}\right)\right|\right)^{2}}{2\left|z-f\left(\theta_{k}^{*}(\theta)\right)\right|^{2}}\right) d \theta\right|,
\end{aligned}
$$

где $\theta_{k}^{*}=\theta_{k}^{*}(\theta)$ - некоторое вещественное число, заключенное между $\theta_{k}$ и $\theta$. Оценим $k$-й член полученной суммы:

$$
\begin{aligned}
& \left|\int_{2 \pi k / n}^{2 \pi(k+1) / n}\left(\frac{\left(|z-f(\theta)|-\left|z-f\left(\theta_{k}\right)\right|\right)}{\left|z-f\left(\theta_{k}\right)\right|}-\frac{\left(|z-f(\theta)|-\left|z-f\left(\theta_{k}\right)\right|\right)^{2}}{2\left|z-f\left(\theta_{k}^{*}(\theta)\right)\right|^{2}}\right) d \theta\right| \\
& \quad \leqslant\left|\int_{2 \pi k / n}^{2 \pi(k+1) / n} \frac{\left(|z-f(\theta)|-\left|z-f\left(\theta_{k}\right)\right|\right)}{\left|z-f\left(\theta_{k}\right)\right|} d \theta\right| \\
& \quad+\left|\int_{2 \pi k / n}^{2 \pi(k+1) / n} \frac{\left(|z-f(\theta)|-\left|z-f\left(\theta_{k}\right)\right|\right)^{2}}{2\left|z-f\left(\theta_{k}^{*}(\theta)\right)\right|^{2}} d \theta\right| .
\end{aligned}
$$

Для функции $|z-f(\theta)|$ переменного $\theta$ имеет место формула Тейлора с остаточньп членом в форме Лагранжа:

$$
|z-f(\theta)|=\left|z-f\left(\theta_{k}\right)\right|+\left|f^{\prime}\left(\hat{\theta}_{k}\right)\right| \cos \left(\arg \left(f\left(\hat{\theta}_{k}\right)-z\right)-\arg f^{\prime}\left(\hat{\theta}_{k}\right)\right)\left(\theta-\theta_{k}\right),
$$

где $\hat{\theta}_{k}=\hat{\theta}_{k}(\theta)$ - некоторое число, лежащее между $\theta$ и $\theta_{k}$. Обозначим через $\log (z-\zeta)$ некоторую однозначную аналитическую по переменной $\zeta$ в окрестности кривой Г ветвь функции $\log (z-\zeta)$. Положим $\left\|f^{\prime}\right\|:=\max \left\{\left|f^{\prime}(\theta)\right|: \theta \in \mathbb{R}\right\}$. Имеем

$$
\begin{aligned}
& \frac{1}{\left|z-f\left(\theta_{k}\right)\right|}\left|\int_{2 \pi k / n}^{2 \pi(k+1) / n}\left(|z-f(\theta)|-\left|z-f\left(\theta_{k}\right)\right|\right) d \theta\right| \\
& =\frac{1}{\left|z-f\left(\theta_{k}\right)\right|}\left|\int_{2 \pi k / n}^{2 \pi(k+1) / n}\right| f^{\prime}\left(\hat{\theta}_{k}\right)\left|\cos \left(\arg \left(f\left(\hat{\theta}_{k}\right)-z\right)-\arg f^{\prime}\left(\hat{\theta}_{k}\right)\right)\left(\theta-\theta_{k}\right) d \theta\right| \\
& =\frac{1}{\left|z-f\left(\theta_{k}\right)\right|}\left|\int_{2 \pi k / n}^{2 \pi(k+1) / n}\right| f^{\prime}\left(\theta_{k}\right) \mid \cos \left(\arg \left(f\left(\theta_{k}\right)-z\right)-\arg f^{\prime}\left(\theta_{k}\right)\right)\left(\theta-\theta_{k}\right) d \theta \\
& +\int_{2 \pi k / n}^{2 \pi(k+1) / n}\left(\left|f^{\prime}\left(\hat{\theta}_{k}\right)\right| \cos \left(\arg \left(f\left(\hat{\theta}_{k}\right)-z\right)-\arg f^{\prime}\left(\hat{\theta}_{k}\right)\right)\right. \\
& \left.-\left|f^{\prime}\left(\theta_{k}\right)\right| \cos \left(\arg \left(f\left(\theta_{k}\right)-z\right)-\arg f^{\prime}\left(\theta_{k}\right)\right)\right)\left(\theta-\theta_{k}\right) d \theta \mid \\
& =\frac{1}{\left|z-f\left(\theta_{k}\right)\right|} \mid \int_{2 \pi k / n}^{2 \pi(k+1) / n}\left(\left|f^{\prime}\left(\hat{\theta}_{k}\right)\right| \cos \left(\arg \left(f\left(\hat{\theta}_{k}\right)-z\right)-\arg f^{\prime}\left(\hat{\theta}_{k}\right)\right)\right. \\
& \left.-\left|f^{\prime}\left(\theta_{k}\right)\right| \cos \left(\arg \left(f\left(\theta_{k}\right)-z\right)-\arg f^{\prime}\left(\theta_{k}\right)\right)\right)\left(\theta-\theta_{k}\right) d \theta \mid \\
& \leqslant \frac{1}{\left|z-f\left(\theta_{k}\right)\right|} \int_{2 \pi k / n}^{2 \pi(k+1) / n}|| f^{\prime}\left(\hat{\theta}_{k}\right) \mid \cos \left(\arg \left(f\left(\hat{\theta}_{k}\right)-z\right)-\arg f^{\prime}\left(\hat{\theta}_{k}\right)\right) \\
& -\left|f^{\prime}\left(\theta_{k}\right)\right| \cos \left(\arg \left(f\left(\theta_{k}\right)-z\right)-\arg f^{\prime}\left(\theta_{k}\right)\right)|| \theta-\theta_{k} \mid d \theta
\end{aligned}
$$




$$
\begin{aligned}
& \leqslant \frac{\pi\left\|f^{\prime}\right\|}{n\left|z-f\left(\theta_{k}\right)\right|} \int_{2 \pi k / n}^{2 \pi(k+1) / n} \mid \cos \left(\arg \left(f\left(\hat{\theta}_{k}\right)-z\right)-\arg f^{\prime}\left(\hat{\theta}_{k}\right)\right) \\
& -\cos \left(\arg \left(f\left(\theta_{k}\right)-z\right)-\arg f^{\prime}\left(\theta_{k}\right)\right) \mid d \theta+\frac{2 \omega\left(f^{\prime}, \mathbb{R} ; \pi / n\right) \pi^{2}}{n^{2}\left|z-f\left(\theta_{k}\right)\right|} \\
& \leqslant \frac{\pi\left\|f^{\prime}\right\|}{n\left|z-f\left(\theta_{k}\right)\right|} \int_{2 \pi k / n}^{2 \pi(k+1) / n}\left|\arg \left(f\left(\hat{\theta}_{k}\right)-z\right)-\arg \left(f\left(\theta_{k}\right)-z\right)\right| \\
& +\left|\arg f^{\prime}\left(\hat{\theta}_{k}\right)-\arg f^{\prime}\left(\theta_{k}\right)\right| d \theta+\frac{2 \omega\left(f^{\prime}, \mathbb{R} ; \pi / n\right) \pi^{2}}{n^{2}\left|z-f\left(\theta_{k}\right)\right|} \\
& \leqslant \frac{\pi\left\|f^{\prime}\right\|}{n\left|z-f\left(\theta_{k}\right)\right|} \int_{2 \pi k / n}^{2 \pi(k+1) / n}\left|\log \left(z-f\left(\hat{\theta}_{k}\right)\right)-\log \left(z-f\left(\theta_{k}\right)\right)\right| d \theta \\
& +\frac{2 \pi^{2}\left(\left\|f^{\prime}\right\| \omega\left(\arg f^{\prime}, \mathbb{R} ; \pi / n\right)+\omega\left(f^{\prime}, \mathbb{R} ; \pi / n\right)\right)}{n^{2}\left|z-f\left(\theta_{k}\right)\right|} \\
& \leqslant \frac{\pi\left\|f^{\prime}\right\|}{n\left|z-f\left(\theta_{k}\right)\right|} \int_{2 \pi k / n}^{2 \pi(k+1) / n} \int_{\theta_{k}}^{\hat{\theta}_{k}} \frac{\left|f^{\prime}(\hat{\theta})\right|}{|z-f(\hat{\theta})|} d \hat{\theta} d \theta \\
& +\frac{2 \pi^{2}\left(\left\|f^{\prime}\right\| \omega\left(\arg f^{\prime}, \mathbb{R} ; \pi / n\right)+\omega\left(f^{\prime}, \mathbb{R} ; \pi / n\right)\right)}{n^{2}\left|z-f\left(\theta_{k}\right)\right|} \\
& \leqslant \frac{\pi^{2}\left\|f^{\prime}\right\|^{2}}{n^{2}\left|\left(z-f\left(\bar{\theta}_{k}\right)\right)\left(z-f\left(\theta_{k}\right)\right)\right|} \int_{2 \pi k / n}^{2 \pi(k+1) / n} 1 d \theta \\
& +\frac{\pi^{2}\left(\left\|f^{\prime}\right\| \omega\left(\arg f^{\prime}, \mathbb{R} ; \pi / n\right)+\omega\left(f^{\prime}, \mathbb{R} ; \pi / n\right)\right)}{n^{2}\left|z-f\left(\theta_{k}\right)\right|}
\end{aligned}
$$

Здесь $\bar{\theta}_{k}=\bar{\theta}_{k}\left(\hat{\theta}_{k}, \theta_{k}\right)$ - некоторое число, лежащее между $\theta_{k}$ и $\hat{\theta}_{k}$.

Пусть некоторое $\theta^{*}$ принадлежит отрезку с концами $\theta_{k}$ и $\theta$, где $\theta \in[2 \pi k / n, 2 \pi(k+1) / n]$. Тогда из наложенного нами ограничения $\rho(z, \Gamma) \geqslant \omega(\varphi, C ; \pi / n)$ следует, что $\left|z-f\left(\theta^{*}\right)\right| \geqslant$ $|z-f(\theta)| / 2$, а следовательно, $1 /\left|z-f\left(\theta^{*}\right)\right| \leqslant 2 /|z-f(\theta)|$. Применим эти неравенства, полагая последовательно $\theta^{*}=\theta_{k}, \theta^{*}=\theta_{k}^{*}$ и $\theta^{*}=\bar{\theta}_{k}$. Отсюда получаем

$$
\begin{gathered}
\frac{\pi}{n\left|z-f\left(\theta_{k}\right)\right|}=\int_{2 \pi k / n}^{2 \pi(k+1) / n} \frac{d \theta}{\left|z-f\left(\theta_{k}\right)\right|} \leqslant 2 \int_{2 \pi k / n}^{2 \pi(k+1) / n} \frac{d \theta}{|z-f(\theta)|}, \\
\int_{2 \pi k / n}^{2 \pi(k+1) / n} \frac{d \theta}{\left|z-f\left(\theta_{k}^{*}\right)\right|^{2}} \leqslant 4 \int_{2 \pi k / n}^{2 \pi(k+1) / n} \frac{d \theta}{|z-f(\theta)|^{2}}, \\
\int_{2 \pi k / n}^{2 \pi(k+1) / n} \frac{d \theta}{\left|\left(z-f\left(\bar{\theta}_{k}\right)\right)\left(z-f\left(\theta_{k}\right)\right)\right|} \leqslant 4 \int_{2 \pi k / n}^{2 \pi(k+1) / n} d \theta|z-f(\theta)|^{2} .
\end{gathered}
$$

Наконец заключаем, что

$$
\begin{aligned}
& \left|\int_{2 \pi k / n}^{2 \pi(k+1) / n} \frac{\left(|z-f(\theta)|-\mid z-f\left(\theta_{k} \mid\right)\right.}{\left|z-f\left(\theta_{k}\right)\right|} d \theta\right| \leqslant 4\left\|f^{\prime}\right\|^{2}\left(\frac{\pi}{n}\right)^{2} \int_{2 \pi k / n}^{2 \pi(k+1) / n} \frac{d \theta}{|z-f(\theta)|^{2}} \\
& \quad+\frac{2 \pi}{n}\left(\left\|f^{\prime}\right\| \omega\left(\arg f^{\prime}, \mathbb{R} ; \frac{\pi}{n}\right)+\omega\left(f^{\prime}, \mathbb{R} ; \frac{\pi}{n}\right)\right) \int_{2 \pi k / n}^{2 \pi(k+1) / n} \frac{d \theta}{|z-f(\theta)|} .
\end{aligned}
$$




\section{Имеем также}

$$
\left|\int_{2 \pi k / n}^{2 \pi(k+1) / n} \frac{\left(|z-f(\theta)|-\left|z-f\left(\theta_{k}\right)\right|\right)^{2}}{2\left|z-f\left(\theta_{k}^{*}(\theta)\right)\right|^{2}} d \theta\right| \leqslant 2 \omega^{2}\left(\varphi, C ; \frac{\pi}{n}\right) \int_{2 \pi k / n}^{2 \pi(k+1) / n} \frac{d \theta}{|z-f(\theta)|^{2}} .
$$

Отсюда получаем

$$
\begin{aligned}
& \left|\int_{2 \pi k / n}^{2 \pi(k+1) / n}\left(\log |z-f(\theta)|-\log \left|z-f\left(\theta_{k}\right)\right|\right) d \theta\right| \\
& \quad \leqslant 2\left(2\left\|f^{\prime}\right\|^{2}\left(\frac{\pi}{n}\right)^{2}+\omega^{2}\left(\varphi, C ; \frac{\pi}{n}\right)\right) \int_{2 \pi k / n}^{2 \pi(k+1) / n} \frac{d \theta}{|z-f(\theta)|^{2}} \\
& \quad+\frac{2 \pi}{n}\left(\left\|f^{\prime}\right\| \omega\left(\arg f^{\prime}, \mathbb{R} ; \frac{\pi}{n}\right)+\omega\left(f^{\prime}, \mathbb{R} ; \frac{\pi}{n}\right)\right) \int_{2 \pi k / n}^{2 \pi(k+1) / n} \frac{d \theta}{|z-f(\theta)|} .
\end{aligned}
$$

Суммирование по $k=0,1, \ldots, n-1$ дает оценку

$$
\begin{aligned}
\sum_{k=1}^{n} \mid & \int_{2 \pi k / n}^{2 \pi(k+1) / n}\left(\log |z-f(\theta)|-\log \left|z-f\left(\theta_{k}\right)\right|\right) d \theta \mid \\
\leqslant & 2\left(2\left\|f^{\prime}\right\|^{2}\left(\frac{\pi}{n}\right)^{2}+\omega^{2}\left(\varphi, C ; \frac{\pi}{n}\right)\right) \int_{0}^{2 \pi} \frac{d \theta}{|z-f(\theta)|^{2}} \\
& +\frac{2 \pi}{n}\left(\left\|f^{\prime}\right\| \omega\left(\arg f^{\prime}, \mathbb{R} ; \frac{\pi}{n}\right)+\omega\left(f^{\prime}, \mathbb{R} ; \frac{\pi}{n}\right)\right) \int_{0}^{2 \pi} \frac{d \theta}{|z-f(\theta)|}
\end{aligned}
$$

Положим $d^{*}:=\rho(z, \Gamma)$. Как было показано при доказательстве теоремы 1 ,

$$
\int_{0}^{2 \pi} \frac{d \theta}{|z-f(\theta)|} \leqslant \pi \int_{d^{*}}^{\infty} \frac{\min \{\omega(\psi ; r), 2\}}{r^{2}} d r
$$

Аналогично показьвается, что

$$
\int_{0}^{2 \pi} \frac{d \theta}{|z-f(\theta)|^{2}} \leqslant 2 \pi \int_{d^{*}}^{\infty} \frac{\min \{\omega(\psi ; r), 2\}}{r^{3}} d r
$$

Окончательно получаем

$$
\begin{aligned}
& \frac{1}{2 \pi} \sum_{k=1}^{n}\left|\int_{2 \pi k / n}^{2 \pi(k+1) / n}\left(\log |z-f(\theta)|-\log \left|z-f\left(\theta_{k}\right)\right|\right) d \theta\right| \\
& \leqslant 2\left(2\left\|f^{\prime}\right\|^{2}\left(\frac{\pi}{n}\right)^{2}+\omega^{2}\left(\varphi, C ; \frac{\pi}{n}\right)\right) \int_{d^{*}}^{\infty} \frac{\min \{\omega(\psi ; r), 2\}}{r^{3}} d r \\
& \quad+\frac{\pi}{n}\left(\left\|f^{\prime}\right\| \omega\left(\arg f^{\prime}, \mathbb{R} ; \frac{\pi}{n}\right)+\omega\left(f^{\prime}, \mathbb{R} ; \frac{\pi}{n}\right)\right) \int_{d^{*}}^{\infty} \frac{\min \{\omega(\psi ; r), 2\}}{r^{2}} d r
\end{aligned}
$$

Как и ранее, рассмотрим многочлен $Q_{n}(z)=\left(\prod_{k=1}^{n}\left(z-a_{k}\right)\right) / c^{n}(\Gamma)$, где $c(\Gamma)=e^{-\gamma}$ $\left(\gamma=\gamma(\Gamma)\right.$ - постоянная Робэна), $a_{k}=f\left(\theta_{k}\right)$. Тогда

$$
\left|\frac{1}{n} \log \right| Q_{n}(z)|-g(z)|=\frac{1}{2 \pi} \sum_{k=1}^{n}\left|\int_{2 \pi k / n}^{2 \pi(k+1) / n}\left(\log |z-f(\theta)|-\log \left|z-f\left(\theta_{k}\right)\right|\right) d \theta\right| .
$$


Повторяя соответствующие рассуждения из доказательства теоремы 1 , получаем, что

$$
\begin{aligned}
\max & \left\{|g(z)|: z \in L\left(Q_{n}, r_{1}\right)\right\} \\
& \leqslant \omega(g ; d)+4\left(2\left\|f^{\prime}\right\|^{2}\left(\frac{\pi}{n}\right)^{2}+\omega\left(\varphi, C ; \frac{\pi}{n}\right)^{2}\right) \int_{d}^{\infty} \frac{\min \{\omega(\psi ; r), 2\}}{r^{3}} d r \\
& +2\left(\left\|f^{\prime}\right\| \omega\left(\arg f^{\prime}, \mathbb{R} ; \frac{\pi}{n}\right)+\omega\left(f^{\prime}, \mathbb{R} ; \frac{\pi}{n}\right)\right)\left(\frac{\pi}{n}\right) \int_{d}^{\infty} \frac{\min \{\omega(\psi ; r), 2\}}{r^{2}} d r
\end{aligned}
$$

для любого $d \geqslant \omega(\varphi, C ; \pi / n)$. Напомним, что это ограничение на параметр $d$ возникло из условия $\rho(z, \Gamma) \geqslant \omega(\varphi, C ; \pi / n)$ на те $z \in G_{\Gamma}$, для которых справедливы изложенные выше рассуждения.

Из условия теоремы следует, что сушествуют такие абсолютные положительные постоянные $C_{1}, C_{2}, C_{3}$, что $\omega(g ; r) \leqslant C_{1} r, \omega(\psi ; r) \leqslant C_{2} r, \omega(\varphi, C ; r) \leqslant C_{3} r$ для любого $r>0$. Тогда

$$
\int_{d}^{\infty} \frac{\min \{\omega(\psi ; r), 2\}}{r^{3}} d r=O\left(\frac{1}{d}\right), \quad \int_{d}^{\infty} \frac{\min \{\omega(\psi ; r), 2\}}{r^{2}} d r=O(-\log d)
$$

$\omega(g ; d)=O(d)$ при $d \rightarrow 0, \omega(\varphi, C ; \pi / n)=O(1 / n)$ при $n \rightarrow \infty$. Положим $d=\omega(\varphi, C ;$ $\pi / n)$. Тогда

$$
\begin{aligned}
& \max \left\{|g(z)|: z \in L\left(Q_{n}, r_{1}\right)\right\} \\
& \quad=O\left(\frac{1}{n}\right)+\left(\left\|f^{\prime}\right\| \omega\left(\arg f^{\prime}, \mathbb{R} ; \frac{\pi}{n}\right)+\omega\left(f^{\prime}, \mathbb{R} ; \frac{\pi}{n}\right)\right) \cdot O\left(\frac{\log n}{n}\right)
\end{aligned}
$$

Используя теперь условия, наложенные на производную $f^{\prime}$ функции $f$, получим

$$
\max \left\{|g(z)|: z \in L\left(Q_{n}, r_{1}\right)\right\}=O\left(\frac{1}{n}\right) \quad \text { при } n \rightarrow \infty .
$$

Докажем, что существует набор чисел $r_{2}=r_{2}(n)>0$ таких, что $\max \{|g(z)|:$ $\left.z \in L\left(\Phi_{n}, r_{2}\right)\right\}=O(1 / n)$. Имеем

$$
\begin{aligned}
\left|\Phi_{n}(z)-\psi^{n}(z)\right|= & \frac{n}{2 \pi}\left|\int_{0}^{2 \pi} \log (f(\theta)-z) e^{i n \theta} d \theta\right|=\frac{1}{2 \pi}\left|\int_{0}^{2 \pi} \frac{f^{\prime}(\theta)}{f(\theta)-z} e^{i n \theta} d \theta\right| \\
= & \frac{1}{4 \pi}\left|\int_{0}^{2 \pi}\left(\frac{f^{\prime}(\theta)}{f(\theta)-z}-\frac{f^{\prime}(\theta-\pi / n)}{f(\theta-\pi / n)-z}\right) e^{i n \theta} d \theta\right| \\
= & \frac{1}{4 \pi} \mid \int_{0}^{2 \pi}\left(f^{\prime}(\theta)\left(\frac{1}{f(\theta)-z}-\frac{1}{f(\theta-\pi / n)-z}\right)\right. \\
& \left.+\frac{1}{f(\theta-\pi / n)-z}\left(f^{\prime}(\theta)-f^{\prime}\left(\theta-\frac{\pi}{n}\right)\right)\right) e^{i n \theta} d \theta \mid \\
= & \frac{1}{4 \pi}\left|\int_{0}^{2 \pi}\left(f^{\prime}(\theta) \frac{-f^{\prime}\left(\theta^{*}\right) \pi}{n\left(f\left(\theta^{*}\right)-z\right)^{2}}+\frac{f^{\prime}(\theta)-f^{\prime}(\theta-\pi / n)}{f(\theta-\pi / n)-z}\right) e^{i n \theta} d \theta\right|,
\end{aligned}
$$


где $\theta^{*}=\theta^{*}(\theta) \in[\theta-\pi / n, \theta]$. Считаем $d^{*}=\rho(z, \Gamma) \geqslant \omega(\varphi, C ; \pi / n)$, тогда $1 /\left|f\left(\theta^{*}\right)-z\right| \leqslant$ $2 /|f(\theta)-z|$. Таким образом,

$$
\begin{aligned}
& \left|\Phi_{n}(z)-\psi^{n}(z)\right| \leqslant \frac{\left\|f^{\prime}\right\|^{2}(\pi / n)}{\pi} \int_{0}^{2 \pi} \frac{d \theta}{|f(\theta)-z|^{2}}+\frac{\omega\left(f^{\prime}, \mathbb{R} ; \pi / n\right)}{2 \pi} \int_{0}^{2 \pi} \frac{d \theta}{|f(\theta)-z|} \\
& \quad \leqslant 2\left\|f^{\prime}\right\|^{2} \frac{\pi}{n} \int_{d^{*}}^{\infty} \frac{\min \{\omega(\psi ; r), 2\}}{r^{3}} d r+\frac{\omega\left(f^{\prime}, \mathbb{R} ; \pi / n\right)}{2} \int_{d^{*}}^{\infty} \frac{\min \{\omega(\psi ; r), 2\}}{r^{2}} d r .
\end{aligned}
$$

Повторяя соответствующие рассуждения из доказательства теоремы 1 , получаем, что

$$
\begin{aligned}
\max \left\{g(z): z \in L\left(\Phi_{n}, r_{2}\right)\right\} \leqslant & \omega\left(g ; d^{*}\right)+\frac{4 \pi\left\|f^{\prime}\right\|^{2}}{n^{2}} \int_{d^{*}}^{\infty} \frac{\min \{\omega(\psi ; r), 2\}}{r^{3}} d r \\
& +\frac{\omega\left(f^{\prime}, \mathbb{R} ; \pi / n\right)}{n} \int_{d^{*}}^{\infty} \frac{\min \{\omega(\psi ; r), 2\}}{r^{2}} d r
\end{aligned}
$$

при $d^{*}=\omega(\varphi, C ; \pi / n)$ и некотором положительном $r_{2}=r_{2}(n)$. Кроме того, лемниската $L\left(\Phi_{n}, r_{2}\right)$ будет замкнутой жордановой кривой, содержащей $\Gamma$ внутри себя. Следовательно,

$$
\max \left\{g(z): z \in L\left(\Phi_{n}, r_{2}\right)\right\}=O\left(\frac{1}{n}\right)+4 \pi\left\|f^{\prime}\right\|^{2} O\left(\frac{1}{n}\right)+\omega\left(f^{\prime}, \mathbb{R} ; \frac{\pi}{n}\right) O\left(\frac{\log n}{n}\right)
$$

при $n \rightarrow \infty$. То есть в условиях теоремы 2 получаем

$$
\max \left\{g(z): z \in L\left(\Phi_{n}, r_{2}\right)\right\}=O\left(\frac{1}{n}\right), \quad n \rightarrow \infty
$$

а следовательно, и $M_{n}(\Gamma)=O(1 / n), n \rightarrow \infty$. По лемме равенство $M_{n}(\Gamma)=O(1 / n)$ эквивалентно $H_{n}(\Gamma)=O(1 / n), n \rightarrow \infty$. Теорема доказана полностью.

СлЕдСТВИЕ 3. Пусть Г - гладкая замкнутая жорданова кривая, угол наклона касательной $\theta(s) \kappa$ которой как функиия длины дуги s имеет модуль непрерывности $\omega(t)$, удовлетворяющий условию

$$
\int_{0}^{\delta} \frac{\omega(t)}{t} d t+\delta \int_{\delta}^{\pi} \frac{\omega(t)}{t^{2}} d t=O\left(\frac{1}{\log (1 / \delta)}\right)
$$

при $\delta \rightarrow 0, \delta>0$. Тогда при $n \rightarrow \infty$ имеют место равенства

$$
H_{n}(\Gamma)=O\left(\frac{1}{n}\right), \quad M_{n}(\Gamma)=O\left(\frac{1}{n}\right)
$$


ДокАЗАтЕльство. Положим $\left\|f^{\prime}\right\|:=\max \left\{\left|f^{\prime}(\theta)\right|: \theta \in \mathbb{R}\right\}$ (напомним, что $f(\theta):=$ $\left.\varphi\left(e^{i \theta}\right)\right)$. Как показал Варшавский (см. [6, теорема 2]), условие $\int_{0}^{\delta} \omega(t) / t d t<\infty$ влечет за собой непрерьвную дифференцируемость функций $\psi$ и $\varphi$ в $\bar{G}_{\Gamma}$ и $\bar{G}_{C}$ соответственно, при этом

$$
\begin{gathered}
\omega_{0}(\delta):=\omega\left(\arg f^{\prime}, \mathbb{R} ; \delta\right) \leqslant \omega\left(\left\|f^{\prime}\right\| \delta\right)+\delta \\
\omega^{*}(\delta):=\omega\left(f^{\prime}, \mathbb{R} ; \delta\right) \leqslant M\left(\int_{0}^{\delta} \frac{\omega_{0}(t)}{t} d t+\delta \int_{\delta}^{\pi} \frac{\omega_{0}(t)}{t^{2}} d t\right)
\end{gathered}
$$

для некоторой абсолютной положительной константы $M, \delta>0$.

Ясно, что

$$
\omega_{0}(\delta) \log \left(\frac{1}{\delta}\right)=\omega_{0}(\delta) \int_{\delta}^{1} \frac{d t}{t} \leqslant \int_{\delta}^{1} \frac{\omega_{0}(t)}{t} d t \leqslant \int_{0}^{1} \frac{\omega_{0}(t)}{t} d t=A<\infty,
$$

где $A>0$ не зависит от $\delta>0$. Следовательно, $\omega_{0}(\delta)=O(1 / \log (1 / \delta))$ при $\delta \rightarrow 0, \delta>0$. Условие же $\omega^{*}(\delta)=O(1 / \log (1 / \delta))$ при $\delta \rightarrow 0$, очевидно, справедливо в силу оценки Варшавского и соотношения (13). По теореме 2 получаем $M_{n}=O(1 / n)$ и $H_{n}=O(1 / n)$ при $n \rightarrow \infty$.

3. ТЕОРемА 3. Пусть Г - линия уровня функиии Грина $g_{K}(z)$ для оператора Лапласа во внешности некоторого континуума $K \subset \mathbb{C}$ со связным дополнением: $\Gamma=\left\{z: g_{K}(z)=\lambda\right\}, \lambda>0$. Тогда

$$
\limsup _{n \rightarrow \infty} \sqrt[n]{H_{n}(\Gamma)}=\limsup _{n \rightarrow \infty} \sqrt[n]{M_{n}(\Gamma)} \leqslant e^{-\lambda}
$$

ДокАЗАТЕЛЬСтво. Напомним, что функция $w=\psi(z)$ конформно и однолистно отображает внешность кривой $\Gamma$ на внешность единичной окружности $C, \psi(\infty)=\infty$, $\psi^{\prime}(\infty)>0, g(z)$ - функция Грина с полюсом в $\infty$ для внешности кривой $Г$. В условиях теоремы функция $\psi(z)$ аналитически продолжается во внешность континуума $K$. Пусть $\varepsilon-$ произвольное число из интервала $(0, \lambda), l:=\left\{z: g_{K}(z)=\varepsilon\right\}$. Очевидно, что $g_{K}(z)-\lambda \equiv g(z)=\log |\psi(z)|$ всюду во внешности континуума $K$. Поэтому при $z \in l$ справедливо равенство $|\psi(z)|=\exp \{-(\lambda-\varepsilon)\}$. Для всех $z \in \bar{G}_{\Gamma} \backslash\{\infty\}$ и некоторого числа $A=A(\varepsilon)>0$ имеем

$$
\left|\Phi_{n}(z)-\psi^{n}(z)\right|=\frac{1}{2 \pi}\left|\int_{l} \frac{\psi^{n}(\zeta)}{\zeta-z} d \zeta\right| \leqslant A \cdot e^{-n(\lambda-\varepsilon)} .
$$

Отсюда, повторяя соответствующие рассуждения из доказательства теоремы 1 , получаем, что

$$
\max \left\{g(z): z \in L\left(\Phi_{n}, r\right)\right\} \leqslant \frac{A}{n} e^{-n(\lambda-\varepsilon)}, \quad \text { где } r:=\max \left\{\left|\Phi_{n}(z)\right|: z \in \Gamma\right\} .
$$

Заметим, что кривая $Г$ лежит в замкнутой области, ограниченной лемнискатой $L=$ $L\left(\Phi_{n}, r\right)$, которая при достаточно больших $n$ является замкнутой аналитической жордановой кривой. Для всех таких $n$ имеем $M_{n}(\Gamma) \leqslant(A / n) e^{-n(\lambda-\varepsilon)}$ и, следовательно, учитьвая произвольность $\varepsilon \in(0, \lambda)$ и лемму, получаем соотношения

$$
\limsup _{n \rightarrow \infty} \sqrt[n]{H_{n}(\Gamma)}=\limsup _{n \rightarrow \infty} \sqrt[n]{M_{n}(\Gamma)} \leqslant e^{-\lambda}
$$


СлЕДСТВИЕ 4. Для любой замкнутой аналитической жордановой кривой Г справедливо неравенство

$$
\limsup _{n \rightarrow \infty} \sqrt[n]{H_{n}(\Gamma)}=\limsup _{n \rightarrow \infty} \sqrt[n]{M_{n}(\Gamma)}<1
$$

ДокАЗАТЕЛЬСТВо. В силу теоремы Шварца об аналитическом продолжении через аналитическую кривую (см., например, [3, т. 2, гл. 8, п. 7.1]) функция $\psi(z)$ аналитически продолжается через Г как однолистная аналитическая функция, конформно отображающая внешность некоторой аналитической жордановой кривой $S$, лежащей строго внутри $\Gamma$, на внешность некоторой окружности $\{z:|z|=R=$ const $<1\}$. Таким образом, кривая Г является линией уровня функции Грина $g_{S}(z)$ для внешности $S$. Отсюда и из теоремы 3 вытекает требуемое.

В заключение хочу искренне поблагодарить профессора Е. П. Долженко за постановки задач и пристальное внимание к работе, а также доцента П. А. Бородина за ценные советы.

\section{СПИСОК ЦИТИРОВАННОЙ ЛИТЕРАТУРЫ}

[1] Hilbert D. Über die Entwicklung einer beliebigen analytischen Funktion einer Variabeln in eine unendliche nach ganzen rationalen Funktionen fortschreitende Reihe // Nachr. Königl. Gesell. Wiss. Göttingen, Math.-Phys. Kl. 1897. P. 63-70.

[2] Голузин Г. М. Геометрическая теория функций комплексного переменного. М.: Наука, 1966.

[3] Маркушевич А. И. Теория аналитических функций. М.: Наука, 1968.

[4] Тамразов П. М. Экстремальные конформные отображения и полюсы квадратичных дифференциалов // Изв. АН СССР. Сер. матем. 1968. Т. 32. № 5. С. 1033-1043.

[5] Долженко Е. П. О конформных отображениях жордановых областей // Вестн. МГУ. Сер. 1. Матем., мех. 1999. №4. С. 66-68.

[6] Warschawski S.E. On differentiability at the boundary in conformal mapping // Proc. Amer. Math. Soc. 1961. V. 12. № 4. P. 614-620. 